\title{
Forecasting ofuseful life of the structures of a production building during operation
}

\author{
Valeriya Pshenichkina ${ }^{1, *}$, Kseniya Suhina ${ }^{1}$, and Artem Zhukov ${ }^{1}$ \\ ${ }^{1}$ Volgograd State Technical University, 400081 Academicheskaya 1, Russia
}

\begin{abstract}
The article presents a probabilistic concept of practical evaluation of the individual resource of structures of industrial buildings and structures in operation. The results of calculation of a monolithic reinforced concrete overpass for safety and durability are given. The quantitative values of the resource are obtained for a given security of operability.
\end{abstract}

\section{Introduction}

At present, there is a certain contradiction in the calculation of structures between the sufficiently high level on which the forces and movements of the system are determined by the methods of structural mechanics, the theory of elasticity, plasticity, and the approximate evaluation of the reliability of buildings and structures in two groups of limiting states only at the beginning of their operation.

The main drawback of the method of limiting states is that it does not take into account the factor of time and wear. These factors are necessary for the establishment of reasonable overhaul periods, assessment of the state of the structures for the purposes of reconstruction, safety assessment and the forecast of the remaining service life. In the deterministic formulation and without taking into account the time factor, it is impossible to solve the main task of calculating structures and bases to provide the required level of reliability throughout the life of the operation.

Quantitative methods for assessing the safety and resource of building objects, which are based on a probabilistic concept, the application of probability models and methods of reliability theory, are not yet widely used in practical studies. This is due to the complexity of carrying out probabilistic calculations that require a large amount of statistical data, the undeveloped engineering techniques. At the same time, the tasks of estimating the residual resource, with sufficient accuracy for practice, can be solved in the correlation approximation, which does not require a significant increase in the volume of information.

\section{Probabilistic concept of durability evaluation}

The rectangu Resource - this is an indicator of longevity, characterizing the reserve of possible operating time of the object. The estimation of the resource of a building is carried out on the basis of theoretical design models taking into account statistical data on loads, impacts, materials and operating conditions of similar buildings or structures. The forecasting of the resource is an integral part of the theory of structural reliability and is of a probabilistic nature, which is due to the lack of complete information about the object and the spread of longevity indicators. Unlike the design stage, resource forecasting at the operational stage is carried out for a particular building or structure, therefore the main resource concept in operation is the individual residual resource or remaining service life-the operating time from this point in time to the limit state. Since in service conditions, between-repair periods are assigned individually according to the technical state of the facility, the concept of an individual resource is used up to the nearest medium or major repair.

Forecasting an individual resource includes a wide range of tasks. At the first stage, an assessment is made of the technical condition of the structure of the facility, the detection of defects and damage by methods of nondestructive testing. The second stage determines the actual load and the actual operating conditions of the structures under study. At the final stage, reconciliation of the actual resource with reliability indicators at the design stage; Prediction of the optimal residual life on the basis of the data obtained, as well as an assessment of the likelihood of failure of structures. In this case, the predicted resource must be coordinated with the designated service life of the building.

Currently, the assessment of the loss of performance of an object, especially for local injuries, is mainly based on a deterministic approach to its output parameters. For load-bearing structures of buildings, the output parameters that determine their operability are the bearing capacity and rigidity, by the variation of which it is possible to judge the course of the process of wear and tear. Assessment of the degree of damage on the output parameters is used in the technical examination of building structures of buildings and structures, as they

\footnotetext{
"Corresponding author: vap hm@list.ru
} 
are influenced by many factors that are difficult to describe.Such factors include: corrosion of steel and reinforced concrete structures, rotting of wooden structures, wetting of structures and associated unfreezing of concrete and masonry, oiling of concrete, fatigue failure of steel structures, alteration of ground conditions for foundations, cracks in reinforced concrete and stone structures, elevated Deformation of structures deflections, death, loss of stability, fluctuations, damage from fire during fires, as well as errors in the design and erection Constructions (human factor) [1]. Diagnostic signs of defects of all types of building structures - ferroconcrete, stone, metal, wooden, and also their bases and foundations, - allow to estimate approximately the category of their technical condition. In this case, the serviceable condition meets the requirements of the design standards, the rules of production and acceptance of construction works. Inoperative condition corresponds to the unsuitable design for operation. Types of characteristic damage and structural defects, as a rule, are reduced to special tables, in which for each defect index, the maximum values are determined in the serviceable state and the minimum values for an inoperable state $[2,3,4]$.

Deterministic evaluation of damages by output parameters approximately establishes the level of reliability of the object at the time of its technical examination, but does not allow to fully determine the causes of the change in output parameters and determine the numerical characteristics of the aging process that occurs in the materials of structures. Such an assessment does not provide an opportunity to obtain a reliable forecast of the residual resource of the building as a whole and its structures. Therefore, the calculation of the residual life of building objects should be carried out using the methods of reliability theory.

Knowledge of the laws of the change in the output parameters of the system makes it possible to calculate the average time of safe operation of the structure, and taking into account the stochastic nature of the process to obtain quantitative values of the risk of failure of the design when the output parameter exceeds the maximum permissible value. Considering the aging processes as random, it is convenient to reduce them to more simple regularities, highlighting the stochastic component in the form of a random variable or a stationary random function. The apparatus of the theory of random functions can be applied as a differential function expressing the speed of the process $v(t)$, and to an integral function describing the change in the degree of damage $u(t)$. But, since it is the speed of the damage process that is determined by the physics of the phenomenon, it is more convenient to use it in computational modeling. Then, the yield parameters $X(t)$ $X(t)=\phi[v(t)][5]$

The law of distribution of service life (operating time) to failure is formed depending on the nature of the course of the wear process in the structures, the level of their initial reliability and safety requirements. However, in practical calculations, from a theoretical distribution laws of random variables known in probability theory, a law is chosen that reflects the statistical regularities of the processes with a high degree of reliability, and can be used to solve the problems of estimating the resource. The basis for choosing the distribution law and its parameters are experimental studies, operational data on the operation of similar objects, theoretical premises. So, with wear and other gradual failures, as a rule, apply a normal law. When external factors that lead to failures, irrespective of its state and duration of previous work, act on the design, that is, when sudden failures occur, they can be described by exponential or uniform distributions $[5,6]$.

It should be noted that direct application of the laws of the distribution of service life can not be recognized as the main for building structures. First, the law is chosen formally, it does not reflect the processes of the formation of a refusal. Secondly, to obtain experimental data that allows us to judge the law of distribution, it takes a long time, since the final stage of damage is fixed-failure.

In [1], devoted to the reliability of machines and structures, V.V. Bolotin considered in general terms all known models of failures, mainly for machine-building structures, which at present it is not possible to use when predicting the resource of building structures during operation due to the lack of sufficient information on the regularities of resource development of various types of structures.

At the same time, the use of instruments and equipment for non-destructive methods for controlling the material of structures (ultrasonic and magnetic equipment, strain gauges, mechanical measuring instruments) allows the processes of accumulation of damages and aging, a decrease in strength and deformation parameters, generalized by means of such a measure of damage as the rate of decrease of the strength parameter. This measure of damage is not directly related to the characteristics of the physical aging processes of the material, but it allows the development of simple and affordable methods for estimating the resource at all stages of the design.

Let's consider a practical method of estimating and forecasting a resource by the example of reinforced concrete structures of an exploited industrial facility. The practice of the operation of structures indicates that the resistance of the elements begins to decrease only after some random time interval $T 1$. The value of $T 1$ is determined experimentally. Then the random lifetime of the construction is $T_{\text {res }}=T 1+T_{\gamma}$, where $T_{\gamma}$ is the operating time of the structure to the limiting state. To illustrate the calculation of the durability of the structure, long-term experimental and estimated estimates of the performance of a monolithic reinforced concrete overpass for rubber mixers at the Voltyrsky tire plant in Volzhsky have been used. The trestle $144 \mathrm{~m}$ long is divided into four blocks by temperaturesedimentary seams. On the platform there are 14 rubber mixers, with the work of which there are dynamic loads. The trestle structure is a frame spatial system with an 
upper structure in the form of a ribbed plate $6.5 \mathrm{~m}$ wide, $0.4 \mathrm{~m}$ thick and a base plate $6.5 \mathrm{~m}$ wide and $0.6 \mathrm{~m}$ thick. Span of the frame $4.25 \mathrm{~m}$, column spacing $6,0 \mathrm{~m}$. Under the project, concrete M-200 (class B15), valves of class A-III (A400). The flyover has been in operation since 1964. In 1980, design mixers were replaced with new, more productive ones. In 1980, a survey was carried out of horizontal and vertical oscillations in a number of sections of the top plate of the overpass and the strength of concrete. It is established that in some zones of the upper plate the amplitudes of the horizontal oscillations in the stationary mode exceed the maximum permissible value $A_{g}=0.1 \mathrm{~mm}$ for machines with rotating parts at $n=750 \mathrm{rpm}$. Mass testing of concrete overpass designs with non-destructive methods showed that there was a decrease in strength, the average statistical value of which was $\bar{R}_{b}=0.130 \cdot 105 \mathrm{kN} /$ $\mathrm{m} 2$. For the beginning of the resource life was adopted in 1980, and the value of $T 1$ in 1996 was 16 years.

Spatial calculation of the trestle for static and dynamic effects allowed to establish the stress-strain state of all structural elements. For an example of calculation for durability, a bolt of one of the intermediate frames of the flyover was considered. Safety of the bolt element at the beginning of operation. The calculated values of $R_{S}, R_{b}, b, h_{0}, M$ were accepted by uncorrelated random variables with the following numerical characteristics: mathematical expectations $\bar{R}_{b}=0.145 \cdot 105 \mathrm{kN} / \mathrm{m} 2$; $\bar{R}_{S}=4.785 \cdot 105 \mathrm{kN} / \mathrm{m} 2 ; \quad \bar{M}=316 \mathrm{kN} \cdot \mathrm{m} ;$ $\bar{h}_{0}=0.66 \mathrm{~m} ; \bar{b}=1.96 \mathrm{~m} ;$ Standards $\sigma_{R_{b}}=1957 \mathrm{kN} /$ $\mathrm{m} 2 ; \sigma_{R_{S}}=4.785 \cdot 104 \mathrm{kN} / \mathrm{m} 2 ; \sigma_{M}=47.4 \mathrm{kN} \cdot \mathrm{m}$; $\sigma_{h_{0}}=0.02 \mathrm{~m} ; \sigma_{b}=0.059 \mathrm{~m}$.

The height of the compressed section of the element $x=\frac{A_{S} \bar{R}_{S}}{\bar{R}_{b} \bar{b}}=0,03 \mathrm{~m}$.

\section{Bearing capacity}

$\bar{\Phi}=\bar{R}_{b} \bar{b} x\left(\bar{h}_{0}-0.5 x\right)=549.34$

of

section mathematical expectation of the function of working capacity [7] $\bar{S}=\bar{\Phi}-\bar{M}=233.34 \quad \mathrm{kN} \cdot \mathrm{m}$. The standard of the health function $\sigma \mathrm{S}$ is determined from expression

$$
\sigma_{S}^{2}=\sum_{1}^{n}\left(\frac{\partial S}{\partial x_{i}}\right)^{2} \sigma_{x_{i}}^{2}
$$

where the function $\mathrm{S}$ is represented in the form

$$
\bar{S}=\bar{R}_{S} A_{S}\left(\bar{h}_{0}-0,5 \frac{\bar{R}_{S} A_{S}}{\bar{R}_{b} \bar{b}}\right)-M
$$

$$
\begin{gathered}
\frac{\partial S}{\partial R_{S}}=A_{S} \bar{h}_{0}-\frac{\bar{R}_{S} A_{S}^{2}}{\bar{R}_{b} \bar{b}} ; \quad \frac{\partial S}{\partial R_{b}}=\frac{A_{S}^{2} \bar{R}_{S}^{2}}{2 \bar{b} \bar{R}_{b}^{2}} ; \\
\frac{\partial S}{\partial h_{0}}=A_{S} \bar{R}_{S} ; \quad \frac{\partial S}{\partial b}=\frac{A_{S}^{2} \bar{R}_{S}^{2}}{2 \bar{R}_{b} \bar{b}^{2}} ; \quad \frac{\partial S}{\partial M}=-1,0 .
\end{gathered}
$$

Denoting the expressions for the partial derivatives by the symbols $a, b, c, d, e$, we obtain the formula for the standard of the health function

$$
\sigma_{S}=\sqrt{\left(a \cdot \sigma_{R_{S}}\right)^{2}+\left(b \cdot \sigma_{R b}\right)^{2}+\left(c \cdot \sigma_{h_{0}}\right)^{2}+\left(d \cdot \sigma_{b}\right)^{2}+\left(e \cdot \sigma_{M}\right)^{2}}=
$$$$
=73.58
$$

Coefficient of variation of the health function

$$
k_{S}=\frac{\sigma_{S}}{\bar{S}}=0,315
$$

Reliability index [8] $\beta=\frac{\bar{\Phi}-\bar{F}}{\sigma_{S}}=\frac{\bar{S}}{\sigma_{S}}=3,17$. The

value of the function of the normalized normal distribution for the obtained reliability index $\Phi(\beta)=0.49924$, then the reliability of the crossbar section will be $P(S)=0.5+0.49924=0.99924$, respectively, the risk is $P_{f}=0.00076$.

The security of the construction is higher than the safety limit $\beta=3.17>3.0$, which characterizes its operability during the whole operation period of the structure.

Evaluation of durability $[9,10,11]$. Indicators durability are gamma $T_{\gamma}$ resource (time between the limit state) and technical resource $T_{\text {res }}=T 1+T_{\gamma}$. The evaluation of the longevity of elements is carried out for a given security of their operability

$$
P\left[T \geq T_{\text {res }}\right]=P\left[T \geq T_{\gamma}\right]=P\left(S_{r}>0\right)=\Phi\left(\beta_{S}\right),
$$

where $\mathrm{r}$ is the final calculated cross section of the process $S(t)$.

The construction of the design for the limit state has been established since 1980 , when $T 1=16$ years, and the strength of the concrete was $\bar{R}_{b}=0.130 \cdot 105 \mathrm{kN} /$ $\mathrm{m}^{2}$. The bearing capacity of the section $\bar{M}=316 \mathrm{kN}$. $\mathrm{m}$ is $\bar{\Phi}_{\mathrm{H}}=\bar{R}_{b} \cdot \bar{b} \cdot x \cdot\left(\bar{h}_{0}-0,5 x\right)=547.98 \mathrm{kN} \cdot \mathrm{m}$. Functionality function $\bar{S}_{\mathrm{H}}=\bar{\Phi}_{\mathrm{H}}-\bar{M}=231.98 \mathrm{kN} \cdot \mathrm{m}$. The standard of the health function $\sigma_{\mathrm{S}_{\mu}}=73.382 \mathrm{kN}$. m. Reliability index $\beta=3.16, \Phi(\beta)=0.4992$, $P(S)=0.9992$.

According to the 1996 data, it was found that: $\bar{R}_{b}=0.105 \cdot 105 \quad \mathrm{kN} \quad / \quad \mathrm{m}^{2} ; \quad \chi=0.041 \quad \mathrm{~m}$; $\bar{\Phi}_{K}=544.48 \quad \mathrm{kN} \quad \cdot \mathrm{m} ; \quad \bar{M}_{K}=350 \mathrm{kN} \quad \cdot \mathrm{m} ;$ $\bar{S}_{K}=194.48 \mathrm{kN} \cdot \mathrm{m} ; \sigma_{S}=72.89 \mathrm{kN} \cdot \mathrm{m} ; \beta=2,67$. 
The wear rate of the section of the element in the time interval $t_{u}=1996-1980$ year $\bar{V}_{R}=\frac{\bar{S}_{\mathrm{H}}-\bar{S}_{\mathrm{K}}}{t_{u}}=2,34$ $\mathrm{kN} \cdot \mathrm{m} /$ year. Function of working capacity of the element with allowance for wear $S(t)=S_{r}-t \cdot V_{r}$, where $S_{r}=S_{H}$.

The reliability index is found from formula

$$
\beta_{S}=\frac{\bar{S}_{r}-t \bar{V}_{R}}{\sqrt{\sigma_{r}^{2}+t^{2} \sigma_{v R}^{2}}} .
$$

We transform this expression

$$
\begin{gathered}
\beta_{S} \sqrt{\sigma_{r}^{2}+t^{2} \sigma_{v R}^{2}}=\bar{S}_{r}-t \bar{V}_{R} . \\
\beta_{S}^{2}\left(\sigma_{r}^{2}+t^{2} \sigma_{v R}^{2}\right)=\overline{\bar{S}}_{r}^{2}-2 \bar{S}_{r} t \bar{V}_{R}+t^{2} \bar{V}_{R}^{2} .
\end{gathered}
$$

We denote: $\quad A=\bar{V}_{R}^{2}-\beta_{S}^{2} \sigma_{v R}^{2} ; \quad B=-2 S_{r} \bar{V}_{R}$; $C=S_{r}^{2}-\beta_{S}^{2} \sigma_{S}^{2}$, then the resource $T_{\gamma}$ for a given security will be

$$
T=\frac{-B-\sqrt{B^{2}-4 A C}}{2 A} .
$$

Define the resource for three cases of system security: $P(S)=0.95, \quad \beta_{\mathrm{S}}=1.64 ; \quad P(S)=0.98, \quad \beta_{\mathrm{S}}=2.06 ;$ $P(S)=0.99, \beta_{\mathrm{S}}=2.34$. The results of the calculation are presented in the table 1 .

Table. 1. The results of the resource calculation

\begin{tabular}{|l|c|c|c|c|c|l|}
\hline$P(S)$ & $\beta_{S}$ & $\mathrm{~A}$ & $\mathrm{~B}$ & $\mathrm{C}$ & $\begin{array}{c}T_{\gamma} \\
\text { (year) }\end{array}$ & $\begin{array}{l}T_{\text {res }} \\
\text { (year) }\end{array}$ \\
\hline 0,95 & 1,64 & 5,16 & -1087 & $3,93 \cdot 104$ & 46,3 & 62,3 \\
\hline 0,98 & 2,06 & 4,97 & -1087 & $3,10 \cdot 104$ & 33,6 & 49,6 \\
\hline 0,99 & 2,34 & 4,82 & -1087 & $2,43 \cdot 104$ & 25,2 & 41,2 \\
\hline
\end{tabular}

Thus, after 5-6 years, counting from 2000, it is necessary to conduct a regular survey of trestle structures and repair and restoration works.

\section{Conclusions}

The above probabilistic methodology allows us to predict the onset of the limiting state in time using only two statistical moments of design parameters: mathematical expectation and variance (standard).

Numerical values of the individual residual life of the operated structures of the production building are obtained by the criterion of the probability of failure-free operation $\mathrm{P}(\mathrm{S})$. This is the rationale for planning the operating mode and preventive measures, leading to an increase in the average life of the structures to be repaired, and the establishment of an optimal service life.

\section{References}

1. V. Bolotin, Mechanical Engineering, 165, 312 p. (1984)

2. A. Dobromyslov, Reference manual., 72 p. (2004)

3. Recommendations for assessing the reliability of building structures by external features. 43 p. (2001)

4. BCH 53-86 (p), Rules for assessing the physical wear of residential buildings. (1986)

5. A. Pronikov, Mechanical Engineering, 592p. (1978)

6. G. Poryvay, Manual for high schools, 384p. (1983)

7. A. Rzhanitsyn, Theory of calculating building structures for reliability, 240 p. (1978)

8. V. Raiser, Scientific publication, 384 p. (2010)

9. V. Pshenichkina, K. Sukhina, State. Architectbuilds.Un-t., 35, 85 p. (2014)

10. V. Pshenichkina, K. Sukhina, Construction mechanics of engineering structures and structures 1(2015).

11. V. Pshenichkina and other, International Review of Civil Engineering 7, pp 158. (2016) 